\title{
A posteriori error estimates of adaptive discontinuous Galerkin methods for monotone quasi-linear elliptic problems
}

\author{
Liming Guo ${ }^{1,}$, Qingmin Zhang ${ }^{2, b}$ \\ ${ }^{1}$ School of Mathematics and Statistics, Xinyang Normal University, Xinyang 464000, China; \\ ${ }^{2}$ School of Tourism, Xinyang Normal University, Xinyang 464000, China. \\ alm@xynu.edu.cn, b zhangqingmina@163.com,
}

Keywords: Incomplete interior penalty Galerkin, A posteriori error estimator, Quasi-linear elliptic problems.

\begin{abstract}
A new residual-based a posteriori error estimator is proposed and analyzed for an incomplete interior penalty Galerkin discretization of monotone quasi-linear elliptic problems. We derive the computable upper and lower bounds on the error measured in an energy norm. It is proved that the estimator may have the same form as the continuous Galerkin finite element methods. Results of numerical example are presented.
\end{abstract}

\section{Introduction}

In the early 1970s, discontinuous Galerkin (DG) methods were firstly introduced to solve the first order hyperbolic equations. Meanwhile, they were applied to the numerical solution of second order elliptic problems. During the past decades, DG methods have been intensively studied due to their flexibility, stability and some other merits. We refer the reader to [2, 4] and the books $[13,18]$.

Our aim is to analyze the error estimator for a discontinuous Galerkin formulation of monotone quasi-linear elliptic problems. In contrast to the a posteriori error estimates for linear elliptic problems (see for example [1, 3, 12, 14, 16, 19] and the reference therein), such work is rare for nonlinear elliptic problems. Indeed, it has been presented in $[5,6,8,10,15]$. The authors of [8] presented a new residual-based reliable a posteriori error estimator for the local discontinuous Galerkin approximations of linear and nonlinear diffusion problems. The authors of [15] considered the a posteriori error analysis of interior penalty hp-version discontinuous Galerkin finite element methods for a class of quasi-linear elliptic problems. Bi and Lin [6] studied the incomplete penalty h-version discontinuous Galerkin methods for monotone nonlinear elliptic problems. Recently, Bi and Ginting [5] proposed and studied the residual-based a posteriori error estimates of symmetric interior penalty discontinuous Galerkin method for solving a class of nonmonotone quasi-linear elliptic problems. Congreve and Houston [10] developed the a priori and a posteriori error analysis of hp-version interior penalty discontinuous Galerkin finite element methods for strongly monotone quasi-Newtonian fluid flows.

In this paper, we focus on the study of error estimator, postponing the convergence analysis of adaptive algorithm to a subsequent work. The error estimator proposed in [6] has three terms. We prove that the last term $\left(\sum_{E \in \mathcal{E}} h_{E}^{-1}\left\|\left[u_{h}\right]\right\|_{0, E}^{2}\right)^{1 / 2}$ is a high-order perturbation. Consequently, the error estimator we proposed is the same as the continuous finite element methods. From [17], one can see that our work in this paper is crucial in the convergence analysis of adaptive discontinuous Galerkin methods. 
The rest of this paper is organized as follows. The model problem and some notations are introduced in Sect. 1. We present the discontinuous Galerkin methods in Sect. 2. The a posteriori error estimates are studied in Sect. 3. The numerical experiment is provided in Sect. 4. Finally, we close with a brief summary of the main results in Sect. 5. Throughout this paper, we use $C$ with or without subscripts to denote a generic positive constant which is independent of $h$ but may depend on $u$. It may take different values at different occurrences.

\section{Preliminaries}

Let $\Omega \in R^{2}$ be an open bounded polygonal domain with Lipschitz boundary $\partial \Omega$. We consider an incomplete interior penalty Galerkin method for the following quasi-linear elliptic boundary value problem:

$$
\begin{cases}-\nabla \cdot \mathbf{A}(x, \nabla u)=f(x) & \text { in } \Omega \\ u=0 & \text { on } \partial \Omega\end{cases}
$$

where $f \in L^{2}(\Omega)$. In this paper, only the case: $\mathbf{A}(x, \nabla u)=a(x,|\nabla u|) \nabla u=\left(A_{1}(x, \nabla u), A_{2}(x, \nabla u)\right)^{T}$ is considered. For simplicity, we suppress the dependence of $\mathbf{A}, a$ on $x$, and write $\mathbf{A}(\nabla u)$, $a(|\nabla u|)$ instead of $\mathbf{A}(x, \nabla u) a(x,|\nabla u|)$.

Remark: In this paper, as in [6], only the homogenous Dirichlet condition is considered. As an outlook, we stress that the results are adapted to cover other types of boundary conditions.

Assume 1.1There exist two constants $C_{0}>0$ and $\alpha>0$, which are independent of $\xi$ and $\eta$ such that

$$
\begin{gathered}
\left|\frac{\partial A_{i}}{\partial \xi_{j}}(\xi)\right| \leq C_{0}, \quad i, j=1,2, \forall \xi \in R^{2} . \\
\sum_{i, j=1}^{2} \frac{\partial A_{i}}{\partial \xi_{j}}(\xi) \eta_{i} \eta_{j} \geq \alpha \sum_{i=1}^{2} \eta_{i}^{2}, \quad \forall \xi, \eta \in R^{2} .
\end{gathered}
$$

For a subdomain $D \subseteq \Omega$, we use the standard notations and definitions for the Sobolev spaces $W^{m, p}(D)$ with norm $\|\cdot\|_{k, p, D}$ and seminorm $\mid \|_{k, p, D}$ (see [9]). To simplify the notation, we drop $p$ if $p=2$, i.e., $\|\cdot\|_{m, 2, D}=\|\cdot\|_{m, D}$. The same convention is also used for the seminorms. When $p=2$, $W^{m, 2}(D)$ is denoted by $H^{m}(D)$. When $m=0, H^{m}(D)$ coincides with $L^{2}(D)$, in this case, the inner product will be denoted by $(\cdot)_{D}$. If $D=\Omega$, let $(\cdot)=(\cdot)_{D}$. We use $\langle u, v\rangle_{E}=\int_{E} u v d s$ for edge integral, then $\|u\|_{0, E}=\langle u, u\rangle_{E}^{1 / 2}$.

Let $\mathcal{T}_{h}(0<h<1)$ be a family of triangulations of the domain $\$ \backslash$ Omega. Moreover, for any $K \in \mathcal{T}_{h}$, let $h_{K}=|K|^{1 / 2}$ and $h=\max \left\{h_{K}: K \in \mathcal{T}_{h}\right\}$. We remark that $\mathcal{T}_{h}$ can be a conforming or nonconforming mesh. Assume $\mathcal{T}_{h}$ satisfies the following conditions (see [17]): The elements of $\mathcal{T}_{h}$ satisfy the minimal angle condition. Moreover, the triangulation $\mathcal{T}_{h}$ is locally quasi-uniform.

For a given positive integer $n$, define the broken Sobolev space associated with $\mathcal{T}_{h}$ by

$$
H^{n}\left(\mathcal{T}_{h}\right)=\left\{v \in L^{2}(\Omega):\left.v\right|_{K} \in H^{n}(K), \forall K \in \mathcal{T}_{h}\right\},
$$

equipped with the broken Sobolev norm and seminorm

When $n=2$, we write $E\left(\mathcal{T}_{h}\right)=H^{2}\left(\mathcal{T}_{h}\right)$. Denote the discontinuous linear finite element space associated with $\mathcal{T}_{h}$ by

$$
V\left(\mathcal{T}_{h}\right)=\left\{v \in L^{2}(\Omega):\left.v\right|_{K} \in P_{1}(K), \forall K \in \mathcal{T}_{h}\right\}
$$


Remark: For the convenience of programming, we use the discontinuous linear finite element space. However, the analysis of this paper can be directly extended to higher order finite element space.

Next, we define the average and the jump operators. Denote by $\mathcal{E}$ the set of all edges in $\mathcal{T}_{h}$. Let $\mathcal{E}=\mathcal{E}_{I} \cup \mathcal{E}_{b}$, where $\mathcal{E}_{I}$ is the set of interior edges and $\mathcal{E}_{b}$ is the set of boundary edges. Let $K^{+}$and $K^{-}$be two adjacent elements in $\mathcal{T}_{h}$ sharing a common edge $E$, and $n_{K}^{ \pm}$be the unit outward vector normal to $\partial K^{ \pm}$, respectively. For given scalar-value function $v$ and vector-value function $\mathbf{q}$ which are smooth inside each element $K^{ \pm}$, define their averages and jumps on $E \in \mathcal{E}_{I}$ by

$\left.\{v\}\right|_{E}=\frac{1}{2}\left(v_{K}^{+}+v_{K}^{-}\right),\left.[v]\right|_{E}=v_{K}^{+} \mathbf{n}_{K}^{+}+v_{K}^{-} \mathbf{n}_{K}^{-},\left.\quad\{\mathbf{q}\}\right|_{E}=\frac{1}{2}\left(\mathbf{q}_{K}^{+}+\mathbf{q}_{K}^{-}\right),\left.\quad[\mathbf{q}]\right|_{E}=\mathbf{q}_{K}^{+} \cdot \mathbf{n}_{K}^{+}+\mathbf{q}_{K}^{-} \cdot \mathbf{n}_{K}^{-}$, where $v_{K}^{+}, \mathbf{q}_{K}^{+}$are $\left.v\right|_{K^{+}},\left.\mathbf{q}\right|_{K^{+}}$and $v_{K}^{-}, \mathbf{q}_{K}^{-}$are $\left.v\right|_{K^{-}},\left.\mathbf{q}\right|_{K^{-}}$. If $E \in \mathcal{E}_{b},\left.\{v\}\right|_{E}=v,\left.\{\mathbf{q}\}\right|_{E}=\mathbf{q}$ and $\left.[v]\right|_{E}=v \mathbf{n},\left.[\mathbf{q}]\right|_{E}=\mathbf{q} \cdot \mathbf{n}$.

\section{Discontinuous Galerkin methods}

Assume that $u_{h}^{G} \in V\left(\mathcal{T}_{h}\right) \cap H_{0}^{1}(\Omega)$ is the solution of the continuous Galerkin finite element method that is given by

$$
\left(\mathbf{A}\left(\nabla u_{h}^{G}\right), \nabla \chi\right)=(f, \chi), \quad \forall \chi \in V\left(\mathcal{T}_{h}\right) \cap H_{0}^{1}(\Omega) .
$$

In order to construct the weak formulation for problem (1), we introduce the form $B_{h}$ defined by

$$
B_{h}(w, v)=\sum_{K \in \mathcal{T}_{h}}(\mathbf{A}(\nabla w), \nabla v)_{K}-\sum_{E \in \mathcal{E}}\langle\{\mathbf{A}(\nabla w)\},[v]\rangle_{E}+\sum_{E \in \mathcal{E}} \gamma h_{E}^{-1}\langle[w],[v]\rangle_{E}, \forall w, v \in E\left(\mathcal{T}_{h}\right),
$$

where $h_{E}=\operatorname{diam}(E)$ and $\gamma$ is the interior penalty parameter independent of $h_{E}$.

Notice that $B_{h}$ is consistent with the equations (1) in the following sense. Assume that $u \in H^{2}(\Omega)$ satisfies (1), then for any $v \in E\left(\mathcal{T}_{h}\right)$, we have

$$
\begin{aligned}
B_{h}(u, v) & =\sum_{K \in \mathcal{T}_{h}}(\mathbf{A}(\nabla u), \nabla v)_{K}-\sum_{\mathrm{E} \in \mathcal{E}}\langle\{\mathbf{A}(\nabla u)\},[v]\rangle_{E} \\
& =-\sum_{K \in \mathcal{T}_{h}}(\nabla \cdot \mathbf{A}(\nabla u), v)_{K}+\sum_{K \in \mathcal{T}_{h}}(\mathbf{A}(\nabla u) \cdot \mathbf{n}, v)_{\partial K}-\sum_{E \in \mathcal{E}}\langle\{\mathbf{A}(\nabla u)\},[v]\rangle_{E} \\
& =(f, v)
\end{aligned}
$$

The incomplete interior penalty discontinuous Galerkin method for (1) is to find $u_{h} \in V\left(\mathcal{T}_{h}\right)$ such that

Therefore,

$$
B_{h}\left(u_{h}, v_{h}\right)=\left(f, v_{h}\right), \quad \forall v_{h} \in V\left(\mathcal{T}_{h}\right) .
$$

$$
B_{h}\left(u, v_{h}\right)=B_{h}\left(u_{h}, v_{h}\right), \quad \forall v_{h} \in V\left(\mathcal{T}_{h}\right) .
$$

The authors of [6] have shown that the equation (7) admits a unique solution if the parameter $\gamma \geq \gamma_{0}\left(\gamma_{0} \geq 1\right)$. Now, we introduce the energy norm which has been defined in [6]

$$
\|\| v \|=\left(|v|_{1, \mathcal{T}_{h}}^{2}+\sum_{E \in \mathcal{E}} \gamma h_{E}^{-1}\|[v]\|_{0, E}^{2}\right)^{1 / 2}
$$

Lemma 2.1[6, 7] For $v \in H^{1}\left(\mathcal{T}_{h}\right)$, there exists a positive constant $C$ independent of $v$ such that

$$
\|v\|_{0, \mathcal{T}_{h}} \leq C\|\| v\|\|
$$

From Lemma 2.1 and the definition of \|\|$\cdot \mid \|$, it is easy to show that $\|v\|_{1, \tau_{h}} \leq C\|\| v \|$. In order to get the a posteriori error estimates of incomplete interior penalty discontinuous Galerkin method, following $[14,17]$, we decompose the discontinuous finite element space $V\left(\mathcal{T}_{h}\right)$ into two subspaces: 
one is the conforming part $V^{c}\left(\mathcal{T}_{h}\right)=V\left(\mathcal{T}_{h}\right) \cap H_{0}^{1}(\Omega)$ and the other is the nonconforming part $V^{n}\left(\mathcal{T}_{h}\right)$. Based on this decomposition, for any $v_{h} \in V\left(\mathcal{T}_{h}\right)$, it can be written as $v_{h}=v_{h}^{c}+v_{h}^{n}$. We recall the estimates on the nonconforming part which have been established in [16] and [17] for conforming and nonconforming meshes.

Lemma 2.2 For each $v_{h} \in V\left(\mathcal{T}_{h}\right)$, there exists a positive constant $C_{1}$ such that

$$
\begin{aligned}
& \left|v_{h}^{n}\right|_{1, \mathcal{T}_{h}}^{2}=\left|v_{h}-v_{h}^{c}\right|_{1, \mathcal{T}_{h}}^{2} \leq C_{1} \sum_{E \in \mathcal{E}} h_{E}^{-1}\left\|\left[v_{h}\right]\right\|_{0, E}^{2}, \\
& \left|v_{h}^{n}\right|_{0, \mathcal{T}_{h}}^{2}=\left|v_{h}-v_{h}^{c}\right|_{0, \mathcal{T}_{h}}^{2} \leq C_{1} \sum_{E \in \mathcal{E}} h_{E}\left\|\left[v_{h}\right]\right\|_{0, E}^{2}
\end{aligned}
$$

\section{A posteriori error estimates}

\subsection{Upper bound}

For any $v_{h} \in V\left(\mathcal{T}_{h}\right)$, denote the element residual on $K \in \mathcal{T}_{h}$ and jump residual on $E \in \mathcal{E}$ by

$$
\left.R\left(v_{h}\right)\right|_{K}=f+\left.\nabla \cdot \mathbf{A}\left(\nabla v_{h}\right)\right|_{K},\left.\quad J\left(v_{h}\right)\right|_{E}=\left.\left[\mathbf{A}\left(\nabla v_{h}\right)\right]\right|_{E} .
$$

In view of the above denotation, define the local a posteriori error estimator by

$$
\eta_{1}^{2}\left(v_{h}, K\right)=h_{K}^{2}\left\|R\left(v_{h}\right)\right\|_{0, K}^{2}+\frac{1}{2} h_{E}\left\|J\left(v_{h}\right)\right\|_{0, \partial K \cap \Omega}^{2}
$$

Then the corresponding global a posteriori error estimator is

$$
\eta_{1}^{2}\left(v_{h}, \mathcal{T}_{h}\right)=\sum_{K \in \mathcal{T}_{h}} \eta_{1}^{2}\left(v_{h}, K\right),
$$

and we write $\eta_{1}\left(u_{h}, \mathcal{T}_{h}\right)=\eta_{1}$. We use the residual-based a posteriori error estimator as the kernel of adaptive incomplete interior penalty Galerkin method. In [6], the authors have proved the following upper bound.

Lemma 3.1 Assume that $u$ and $u_{h}$ are the solutions of (1) and (7), respectively. Then we have

$$
\left\|\nabla\left(u-u_{h}\right)\right\|_{0, \mathcal{T}_{h}} \leq C \eta_{2},
$$

where $\eta_{2}=\eta_{1}+\left(\sum_{E \in \mathcal{E}} h_{E}^{-1}\left\|\left[u_{h}\right]\right\|_{0, E}^{2}\right)^{1 / 2}$.

In the rest of this section, we prove that $\sum_{E \in \mathcal{E}} h_{E}^{-1}\left\|\left[u_{h}\right]\right\|_{0, E}^{2}$ is a high order perturbation. Therefore, $\eta_{1}$ can be viewed as a posteriori error estimator that is our main result.

Theorem 3.1 Assume that $u, u_{h}$ and $u_{h}^{G}$ are the solutions of (1), (7) and (4), respectively. If $\gamma \geq \gamma_{1}>\gamma_{0}$, we have

$$
\sum_{K \in \mathcal{T}_{h}}\left\|\nabla\left(u-u_{h}^{G}\right)\right\|_{0, K}^{2} \leq \frac{C_{4}}{\gamma} \eta_{1}^{2}, \quad \sum_{E \in \mathcal{E}} h_{E}^{-1}\left\|\left[u_{h}\right]\right\|_{0, E}^{2} \leq \frac{C_{4}}{\gamma} \eta_{1}^{2}
$$

Proof For any $\chi \in V\left(\mathcal{T}_{h}\right) \cap H_{0}^{1}(\Omega)$, we have

$$
B_{h}(u, \chi)=(f, \chi), \quad B_{h}\left(u_{h}, \chi\right)=(f, \chi), \quad B_{h}\left(u_{h}^{G}, \chi\right)=(f, \chi) .
$$

Let $\beta=\frac{1}{2} \min \{\alpha, 1\}$, it then follows from Lemma 3.3 of [6] that

$$
\beta\left|\left\|u_{h}-u_{h}^{G}\right\|\right| \leq B_{h}\left(u_{h}, u_{h}-u_{h}^{G}\right)-B_{h}\left(u_{h}^{G}, u_{h}-u_{h}^{G}\right)
$$

Using (8) and (11), the right hand side of (12) can be rewritten as

$$
\begin{aligned}
B_{h}\left(u_{h}, u_{h}-u_{h}^{G}\right)-B_{h}\left(u_{h}^{G}, u_{h}-u_{h}^{G}\right)= & B_{h}\left(u_{h}, u_{h}-u_{h}^{G}-\chi\right)-B_{h}\left(u_{h}^{G}, u_{h}-u_{h}^{G}-\chi\right) \\
= & \sum_{K \in \mathcal{T}_{h}}\left(\mathbf{A}(\nabla u)-\mathbf{A}\left(\nabla u_{h}\right)+\mathbf{A}\left(\nabla u_{h}\right)-\mathbf{A}\left(\nabla u_{h}^{G}\right), \nabla\left(u_{h}-u_{h}^{G}-\chi\right)\right)_{K} \\
& -\sum_{E \in \mathcal{E}}\left\langle\left\{\mathbf{A}(\nabla u)-\mathbf{A}\left(\nabla u_{h}\right)+\mathbf{A}\left(\nabla u_{h}\right)-\mathbf{A}\left(\nabla u_{h}^{G}\right)\right\},\left[u_{h}\right]\right\rangle_{E} .
\end{aligned}
$$


Then by Green's formula, we have

$$
\begin{aligned}
& \sum_{K \in \mathcal{T}_{h}}\left(-\nabla \cdot \mathbf{A}(\nabla u)+\nabla \cdot \mathbf{A}\left(\nabla u_{h}\right), u_{h}-u_{h}^{G}-\chi\right)_{K}+\sum_{K \in \mathcal{T}_{h}} \int_{\partial K} \mathbf{A}(\nabla u)-\mathbf{A}\left(\nabla u_{h}\right) \cdot \mathbf{n}\left(u_{h}-u_{h}^{G}-\chi\right) d s \\
& -\sum_{E \in \mathcal{E}}\left\langle\left\{\mathbf{A}(\nabla u)-\mathbf{A}\left(\nabla u_{h}\right)\right\},\left[u_{h}\right]\right\rangle_{E}+I_{3}+I_{4} \\
= & \sum_{K \in \mathcal{T}_{h}}\left(R\left(u_{h}\right), u_{h}-u_{h}^{G}-\chi\right)_{K}-\sum_{E \in \mathcal{E}_{I}}\left\langle\left\{\mathbf{A}(\nabla u)-\mathbf{A}\left(\nabla u_{h}\right)\right\},\left[u_{h}\right]\right\rangle_{E}+I_{3}+I_{4} \\
= & I_{1}+I_{2}+I_{3}+I_{4},
\end{aligned}
$$

where

$$
\begin{gathered}
I_{1}=\sum_{K \in \mathcal{T}_{h}}\left(R\left(u_{h}\right), u_{h}-u_{h}^{G}-\chi\right)_{K}, I_{2}=\sum_{E \in \mathcal{E}_{1}}\left\langle J\left(u_{h}\right),\left[u_{h}\right]\right\rangle_{E}, \\
I_{3}=\sum_{K \in \mathcal{T}_{h}}\left(\mathbf{A}\left(\nabla u_{h}\right)-\mathbf{A}\left(\nabla u_{h}^{G}\right), \nabla\left(u_{h}-u_{h}^{G}-\chi\right)\right)_{K}, I_{4}=-\sum_{E \in \mathcal{E}}\left\langle\left\{\mathbf{A}\left(\nabla u_{h}\right)-\mathbf{A}\left(\nabla u_{h}^{G}\right)\right\},\left[u_{h}\right]\right\rangle_{E}
\end{gathered}
$$

Next, we bound the terms on the right hand of (14) separately. Using the Cauchy--Schwarz inequality and the Young's inequality, for any $\varepsilon_{1}>0$, we have

$$
\begin{aligned}
& I_{1} \leq \frac{1}{2 \varepsilon_{1} \gamma} \sum_{K \in \mathcal{T}_{h}} h_{K}^{2}\left\|R\left(u_{h}\right)\right\|_{0, K}^{2}+\frac{\varepsilon_{1} \gamma}{2} \sum_{K \in \mathcal{T}_{h}} h_{K}^{-2}\left\|u_{h}-u_{h}^{G}-\chi\right\|_{0, K}^{2} \\
& I_{2} \leq \frac{1}{2 \varepsilon_{1} \gamma} \sum_{E \in \mathcal{E}_{I}} h_{E}\left\|J\left(u_{h}\right)\right\|_{0, E}^{2}+\frac{\varepsilon_{1} \gamma}{2} \sum_{E \in \mathcal{E}_{I}} h_{E}^{-1}\left\|\left\{u_{h}-u_{h}^{G}-\chi\right\}\right\|_{0, E}^{2}
\end{aligned}
$$

For any $\varepsilon_{2}>0$, the same arguments and (2) lead to

$$
\begin{aligned}
I_{3} \leq & 2 C_{0} \sum_{K \in \mathcal{T}_{h}}\left\|\nabla\left(u_{h}-u_{h}^{G}\right)\right\|_{0, K}\left\|\nabla\left(u_{h}-u_{h}^{G}-\chi\right)\right\|_{0, K} \leq C_{0} \varepsilon_{2} \sum_{K \in \mathcal{T}_{h}}\left\|\nabla\left(u_{h}-u_{h}^{G}\right)\right\|_{0, K}^{2}+\frac{C_{0}}{\varepsilon_{2}} \sum_{K \in \mathcal{T}_{h}}\left|u_{h}-u_{h}^{G}-\chi\right|_{1, K}^{2} .(17 \\
I_{4} & =\sum_{E \in \mathcal{E}} h_{E}^{1 / 2}\left\|\left\{\mathbf{A}\left(\nabla u_{h}\right)-\mathbf{A}\left(\nabla u_{h}^{G}\right)\right\}\right\|_{0, E} \cdot h_{E}^{-1 / 2}\left\|\left[u_{h}\right]\right\|_{0, E} \leq \frac{\varepsilon_{2}}{2} \sum_{K \in \mathcal{T}_{h}}\left\|\mathbf{A}\left(\nabla u_{h}\right)-\mathbf{A}\left(\nabla u_{h}^{G}\right)\right\|_{0, \partial K}^{2}+\frac{1}{2 \varepsilon_{2}} \sum_{E \in \mathcal{E}} h_{E}^{-1}\left\|\left[u_{h}\right]\right\|_{0, E}^{2} \\
& \leq \frac{C \varepsilon_{2}}{2} \sum_{K \in \mathcal{T}_{h}} h_{E}\left\|\nabla\left(u_{h}-u_{h}^{G}\right)\right\|_{0, \partial K}^{2}+\frac{1}{2 \varepsilon_{2}} \sum_{E \in \mathcal{E}} h_{E}^{-1}\left\|\left[u_{h}\right]\right\|_{0, E}^{2} .
\end{aligned}
$$

Then, it follows from the scaled trace inequality $\|w\|_{0, E}^{2} \leq C\left(h_{E}^{-1}\|w\|_{0, K}^{2}+h_{E}\|\nabla w\|_{0, K}^{2}\right), \forall w \in H^{1}(K)$ and the inverse inequality that

$$
I_{4} \leq \frac{C \varepsilon_{2}}{2} \sum_{K \in \mathcal{T}_{h}}\left\|\nabla\left(u_{h}-u_{h}^{G}\right)\right\|_{0, K}^{2}+\frac{1}{2 \varepsilon_{2}} \sum_{E \in \mathcal{E}} h_{E}^{-1}\left\|\left[u_{h}\right]\right\|_{0, E}^{2} .
$$

Let $\chi$ be the conforming part of $u_{h}-u_{h}^{G}$, from Lemma 2.2, we have

$$
\begin{gathered}
\sum_{K \in \mathcal{T}_{h}} h_{K}^{-2}\left\|u_{h}-u_{h}^{G}-\chi\right\|_{0, K}^{2} \leq C_{1} \sum_{E \in \mathcal{E}} h_{E}^{-1}\left\|\left[u_{h}\right]\right\|_{0, E}^{2} . \\
\sum_{K \in \mathcal{T}_{h}}\left\|\nabla\left(u_{h}-u_{h}^{G}-\chi\right)\right\|_{0, K}^{2} \leq C_{1} \sum_{E \in \mathcal{E}} h_{E}^{-1}\left\|\left[u_{h}\right]\right\|_{0, E}^{2} .
\end{gathered}
$$

Therefore

$$
\sum_{E \in \mathcal{E}_{I}} h_{E}^{-1}\left\|\left\{u_{h}-u_{h}^{G}-\chi\right\}\right\|_{0, E}^{2} \leq C_{3} \sum_{K \in \mathcal{T}_{h}} h_{K}^{-2}\left\|u_{h}-u_{h}^{G}-\chi\right\|_{0, K}^{2} \leq C_{3} C_{1} \sum_{E \in \mathcal{E}} h_{E}^{-1}\left\|\left[u_{h}\right]\right\|_{0, E}^{2} .
$$

Putting (20), (21) and (22) into (15), (16) and (17), we get

$$
\begin{aligned}
\beta\|\| u_{h}-u_{h}^{G}\left\||\leq| I_{1}|+| I_{2}|+| I_{3}|+| I_{4} \mid \leq \frac{1}{2 \varepsilon_{1} \gamma} \sum_{K \in \mathcal{T}_{h}} h_{K}^{2}\right\| R\left(u_{h}\right)\left\|_{0, K}^{2}+\frac{\varepsilon_{1} \gamma C_{1}}{2} \sum_{E \in \mathcal{E}} h_{E}^{-1}\right\|\left[u_{h}\right] \|_{0, E}^{2} \\
\quad+\frac{1}{2 \varepsilon_{1} \gamma} \sum_{E \in \mathcal{E}_{I}} h_{E}\left\|J\left(u_{h}\right)\right\|_{0, E}^{2}+\frac{\varepsilon_{1} \gamma C_{3} C_{1}}{2} \sum_{E \in \mathcal{E}} h_{E}^{-1}\left\|\left[u_{h}\right]\right\|_{0, E}^{2}+C_{0} \varepsilon_{2} \sum_{K \in \mathcal{T}_{h}}\left\|\nabla\left(u_{h}-u_{h}^{G}\right)\right\|_{0, K}^{2}
\end{aligned}
$$




$$
+\frac{C_{0} C_{1}}{\varepsilon_{2}} \sum_{E \in \mathcal{E}} h_{E}^{-1}\left\|\left[u_{h}\right]\right\|_{0, E}^{2}+\frac{C_{2} \varepsilon_{2}}{2} \sum_{K \in \mathcal{T}_{h}}\left\|\nabla\left(u_{h}-u_{h}^{G}\right)\right\|_{0, K}^{2}+\frac{1}{2 \varepsilon_{2}} \sum_{E \in \mathcal{E}} h_{E}^{-1}\left\|\left[u_{h}\right]\right\|_{0, E}^{2} .
$$

According to the definition of energy norm $|\|\cdot\|| \|$, the above inequality can be rewritten as

$$
\begin{aligned}
& \left(\beta-\varepsilon_{2}\left(C_{0}+\frac{C_{2}}{2}\right)\right) \sum_{K \in \mathcal{T}_{h}}\left\|\nabla\left(u_{h}-u_{h}^{G}\right)\right\|_{0, K}^{2}+\left(\beta \gamma-\frac{\varepsilon_{1} C_{1}\left(C_{3}+1\right) \gamma}{2}-\frac{C_{0} C_{1}}{\varepsilon_{2}}-\frac{1}{2 \varepsilon_{2}}\right) \sum_{E \in \mathcal{E}} h_{E}^{-1}\left\|\left[u_{h}\right]\right\|_{0, E}^{2} \\
& \leq \frac{1}{2 \varepsilon_{1} \gamma}\left(\sum_{K \in \mathcal{T}_{h}} h_{K}^{2}\left\|R\left(u_{h}\right)\right\|_{0, K}^{2}+\sum_{E \in \mathcal{E}_{I}} h_{E}\left\|J\left(u_{h}\right)\right\|_{0, E}^{2}\right)
\end{aligned}
$$

Choose sufficiently small $\varepsilon_{1}, \varepsilon_{2}$ such that

$$
\frac{\beta}{6} \leq \frac{\varepsilon_{1} C_{1}\left(C_{3}+1\right)}{2} \leq \frac{\beta}{4}, \quad \varepsilon_{2}\left(C_{0}+\frac{C_{2}}{2}\right) \leq \frac{\beta}{2}
$$

Let $\gamma_{1}=\frac{2\left(2 C_{0} C_{1}+1\right)}{\varepsilon_{2} \beta}$, when $\gamma \geq \gamma_{1}$, we have

$$
\beta \gamma-\frac{\varepsilon_{1} C_{1}\left(C_{3}+1\right) \gamma}{2}-\frac{C_{0} C_{1}}{\varepsilon_{2}}-\frac{1}{2 \varepsilon_{2}} \geq \frac{\beta \gamma}{2}
$$

Then

$$
\begin{aligned}
\frac{\beta}{2} \sum_{K \in \mathcal{T}_{h}}\left\|\nabla\left(u_{h}-u_{h}^{G}\right)\right\|_{0, K}^{2}+\frac{\beta \gamma}{2} \sum_{E \in \mathcal{E}} h_{E}^{-1}\left\|\left[u_{h}\right]\right\|_{0, E}^{2} & \leq \frac{1}{2 \varepsilon_{1} \gamma}\left(\sum_{K \in \mathcal{T}_{h}} h_{K}^{2}\left\|R\left(u_{h}\right)\right\|_{0, K}^{2}+\sum_{E \in \mathcal{E}_{I}} h_{E}\left\|J\left(u_{h}\right)\right\|_{0, E}^{2}\right) \\
& \leq \frac{3 C_{1}\left(C_{3}+1\right)}{2 \beta \gamma} \eta_{1}^{2}
\end{aligned}
$$

Thus, we get the desired results with $C_{4}=\frac{3 C_{1}\left(C_{3}+1\right)}{\beta^{2}}$.

Corollary Assume that $u$ and $u_{h}$ are the solutions of (1) and (7), respectively. If $\gamma \geq \gamma_{1}>\gamma_{0}$, we have

$$
\left\|u-u_{h}\right\| \leq C \eta_{1} .
$$

Proof By Lemma 3.1 and Theorem 3.1, we have

$$
\begin{aligned}
\left\|u-u_{h}\right\| & \leq\left\|\nabla\left(u-u_{h}\right)\right\|_{0, T_{h}}+\left(\sum_{E \in \mathcal{E}} \gamma h_{E}^{-1}\left\|\left[u_{h}\right]\right\|_{0, E}^{2}\right)^{1 / 2} \\
& \leq C\left(\eta_{1}+\left(\sum_{E \in \mathcal{E}} h_{E}^{-1}\left\|\left[u_{h}\right]\right\|_{0, E}^{2}\right)^{1 / 2}\right)+\left(\sum_{E \in \mathcal{E}} \gamma h_{E}^{-1}\left\|\left[u_{h}\right]\right\|_{0, E}^{2}\right)^{1 / 2} \leq C\left(1+\frac{1}{\gamma}+\frac{1}{\gamma^{1 / 2}}\right) \eta_{1} \leq C \eta_{1} .
\end{aligned}
$$

\subsection{Local lower bound}

We introduce the oscillations of $R\left(u_{h}\right)$ and $J\left(u_{h}\right)$ by

$$
\operatorname{osC}_{R, K}^{2}\left(u_{h}\right)=h_{K}^{2}\left\|R\left(u_{h}\right)-\overline{R\left(u_{h}\right)}\right\|_{0, K}^{2}, o s C_{J, E}^{2}\left(u_{h}\right)=h_{E}\left\|J\left(u_{h}\right)-\overline{J\left(u_{h}\right)}\right\|_{0, E}^{2}
$$

where $\overline{R\left(u_{h}\right)}$ is the average value of $R\left(u_{h}\right)$ over the element $K$ and $\overline{J\left(u_{h}\right)}$ is the average value of $J\left(u_{h}\right)$ over the edge $E$. The following theorem can be derived from Theorem 4.7 of [6], we omit its proof.

Theorem 3.2 Assume that $u$ and $u_{h}$ are the solutions of (1) and (7), respectively. Then we have

$$
\eta_{1}\left(u_{h}, K\right) \leq C\left(\sum_{K \in \omega_{K}}\left(\left\|u-u_{h}\right\|_{1, K}^{2}+o s C_{R, K}^{2}\left(u_{h}\right)\right)+\sum_{E \in \partial K} o s C_{J, E}^{2}\left(u_{h}\right)\right)^{1 / 2},
$$

where $\omega_{K}$ is the patch of elements sharing at least a common edge with $K$.

\section{Numerical example}

In order to illustrate the theoretical results, two types of the a posteriori error estimators are considered. The nonlinear system of algebraic equations are solved with the aid of Newton iteration. 
Denote the degrees of freedom by DOFs. Define $E f f_{1}=\eta_{1} /\left\|u-u_{h}\right\|\left|, E f f_{1}=\eta_{2} /\left\|u-u_{h}\right\|\right|$ which are so-called effectivity indices.

Let us consider the problem (1) with $\Omega=(0,1)^{2}$ and $\mathbf{A}(\nabla u)=\left(1+e^{-|\nabla u|^{2}}\right) \nabla u$. We choose $f(x)$ such that the analytical solution $u$ is

$$
u(x, y)=\left(x-x^{2}\right)\left(y-y^{2}\right) \arctan \left(60 \sqrt{(x-1.25)^{2}+(y+0.25)^{2}}-60\right) .
$$

In this numerical problem, we choose Dörfler strategy [11] and set $\theta=0.5$. For the mesh refinement method, we use the newest vertex bisection. The left of Fig. 1 and Fig. 2 show the meshes generated by adaptive algorithm based on error estimators $\eta_{1}$ and $\eta_{2}$, respectively. The right of Fig. 1 and Fig. 2 show convergence history and effectivity indices based on error estimators $\eta_{1}$ and $\eta_{2}$, respectively.
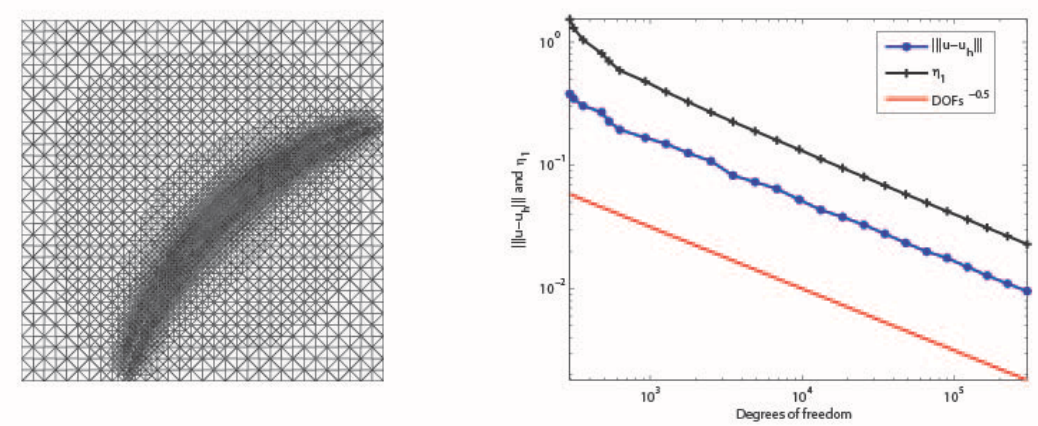

Fig.1 AFEM based on $\eta_{1}$ (Left) Mesh after 18 adaptive steps with 11828 elements; (Right) Convergence history of adaptive algorithm in log-log coordinates.
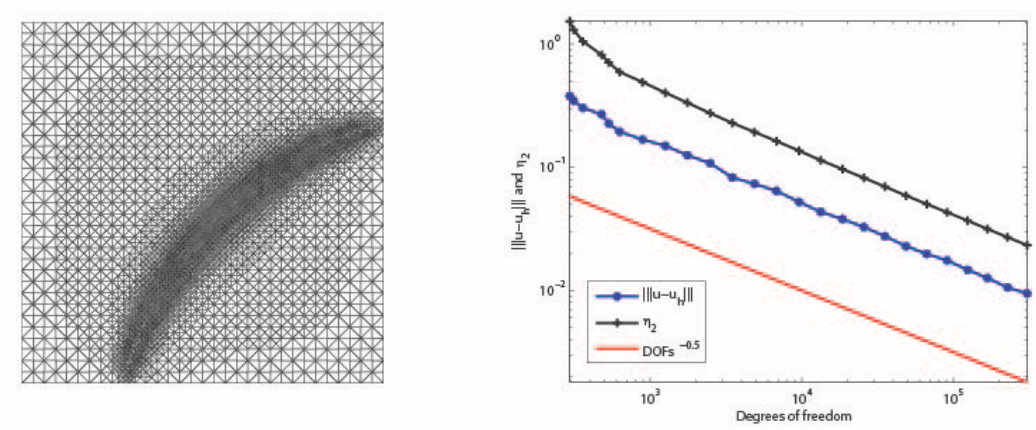

Fig.2 AFEM based on $\eta_{2}$ (Left) Mesh after 18 adaptive steps with 11944 elements; (Right) Convergence history of adaptive algorithm in log-log coordinates.

We can see from Fig. 1 and Fig. 2 that:

1. Both error estimators have accurately detected the singularities of the solution $u$ which are along the circular curve in the domain $\Omega$, but adaptive algorithm based on $\eta_{2}$ refines slightly more elements than that based on $\eta_{1}$.

2. Both adaptive finite element methods get an optimal rate of convergence $\left\|u-u_{h}\right\| \| \approx\left(D O F s^{-1 / 2}\right)$. The two error estimators are both effective since the effectivity indices are bounded from below and above.

\section{Conclusions}

In this paper, we considered a new residual-based a posteriori error estimator for an incomplete interior penalty Galerkin discretization of second order quasi-linear elliptic problems. The computable upper and lower bounds on the error in an energy norm were derived. Numerical results confirmed our theoretical findings. 
From the numerical results, it can be seen that both adaptive algorithms are available because both of them get the optimal convergence rate $\left\|\mid u-u_{h}\right\| \| O\left(D O F s^{-1 / 2}\right)$ and the effectivity indices are bounded. The two error estimators accurately detected the singularities of the solution.

\section{Acknowledgments}

This work is supported by Nanhu Scholars Program for Young Scholars of XYNU and Doctoral Scientific Research Startup Fund of Xinyang Normal University(15021).

\section{References}

[1] M. Ainsworth, A posteriori error estimation for discontinuous Galerkin finite element approximation, SIAM J. Numer. Anal. 45 (2007) 1777-1798.

[2] D. Arnold, F. Brezzi, B. Cockburn, L. Marini, Unified analysis of discontinuous Galerkin methods for elliptic problems, SIAM J. Numer. Anal. 39(2002), 1749-1779.

[3] R. Becker, P. Hansbo, M. G. Larson, Energy norm a posteriori error estimation for discontinuous Galerkin methods, Comput. Methods Appl. Mech. Engrg. 192(2003) 723-733.

[4] F. Brezzi , G. Manzini, D. Marini, P. Pietra, A. Russo, Discontinuous Galerkin approximations for elliptic problems, Numer. Methods Partial Differ. Equ. 16 (2000) 365-378.

[5] C. Bi, V. Ginting, A posteriori error estimates of discontinuous Galerkin method for nonmonotone quasi-linear elliptic problems, J. Sci. Comput. 55(2013) 659-687.

[6] C. Bi, Y. Lin, Discontinuous Galerkin method for monotone nonlinear elliptic problems, Int. J. Numer. Anal. Mod. 9(2012) 999-1024.

[7] S. Brenner, Poincaré-friedrichs inequalities for piecewise $H^{1}$ functions, SIAM J. Numer. Anal. 41(2003) 306-324.

[8] R. Bustinza, G. N.Gatica, B.Cockburn, An a posteriori error estimate for the local discontinuous Galerkin method applied to linear and nonlinear diffusion problems, J. Sci. Comput. 22(2005) 147-185.

[9] P. G. Ciarlet, The Finite Element Method for Elliptic Problems, North-Holland, Amsterdam, 1978.

[10] S. Congreve, P. Houston, E. Suli, and T. P. Wihler, Discontinuous Galerkin finite element approximation of quasilinear elliptic boundary value problems ii: Strongly monotone

quasi-newtonian flows, IMA J. Numer. Anal. 33(2013) 1386-1415.

[11] W. Dörfler, A convergent adaptive algorithm for Poisson's equation, SIAM J. Numer. Anal. 33, 1106-1124 (1996).

[12] A. Ern, A. F. Stephansen, M. Vohralík, Guaranteed and robust discontinuous Galerkin a posteriori error estimates for convection-diffusion-reaction problems, J. Comput. Appl. Math. 234(2010) 114-130.

[13] J. S. Hesthaven, T. Warburton, Nodal discontinuous Galerkin methods: algorithms, analysis, and applications. Springer-Verlag, New York, 2008.

[14] P. Houston, D. Schötzau, T. P. Wihler, Energy norm a posteriori error estimation of hp-adaptive discontinuous Galerkin methods for elliptic problems, Math. Models Methods Appl. Sci. 17(2007), 33-62.

[15] P. Houston, E. Süli, T. P. Wihler, A posteriori error analysis of hp-version discontinuous Galerkin finite-element methods for second-order quasi-linear elliptic PDEs, IMA J. Numer. Anal. 28(2008), 245-273.

[16] O. A. Karakashian, F. Pascal, A posteriori error estimates for a discontinuous Galerkin approximation of second-order elliptic problems, SIAM J. Numer. Anal. 41(2003) 2374-2399.

[17] O. A. Karakashian, F. Pascal, Convergence of adaptive discontinuous Galerkin approximations of second-order elliptic problems, SIAM J. Numer. Anal. 45(2007), 641-665.

[18] B. Rivière, Discontinuous Galerkin Methods for Solving Elliptic and Parabolic Equations: Theory and Implementation, SIAM, 2008. 
[19] D. Schötzau, L. Zhu, A robust a-posteriori error estimator for discontinuous Galerkin methods for convection-diffusion equations, Appl. Numer. Math. 59(2009) 2236-2255. 